\title{
Differentiation and Attraction in Constructional Change
}

Welcome back to these Ten Lectures on Diachronic Construction Grammar. In my last lecture yesterday, I have gone into the topic of what happens diachronically with constructions that can be seen as alternatives to each other. In certain cases, constructions are in mutual competition, and over time, one member of an alternating pair of constructions can replace another one.

I have said that in cases of this kind, we typically have variation that includes social factors. I have mentioned that Bill Croft (2000: 166) has argued for a view of propagation that is socially motivated, so that language-external factors play an important role. Today, I will be looking at another phenomenon in which several constructions act as alternatives to each other. The topic for this lecture is "differentiation and attraction in constructional change". We'll be looking at a set of constructions that stand in a paradigmatic relation and that speakers can choose from for the expression of a given idea. We will see how these constructions develop over time.

In contrast to yesterday, this will not be a story of one construction winning out over another one. Rather, we will see that some constructions become more different from one another, and others are attracted to one another and become more similar. As in the previous studies before, it is very useful to think about these developments in terms of links between constructions. On the node-centered view of constructions that still prevails in the field, we would simply say that over time there is change in the features of the constructions that are inscribed in the nodes. As two constructions are differentiating more and more, their features become maximally different over time. That is a valid way of thinking about it, but I would like to suggest that there is a much more natural way to think about it, namely in terms of links.

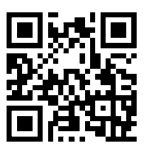

All original audio-recordings and other supplementary material, such as any hand-outs and powerpoint presentations for the lecture series, have been made available online and are referenced via unique Dor numbers on the website www.figshare.com. They may be accessed via this QR code and the following dynamic link: https://doi.org/10.6o84/mg.figshare.13691182 
Let's say that there are two forms, A and B, and each of them is connected with a range of meanings. Some of these meanings overlap. There are some meanings that are specific to A, and some meanings that are specific to $\mathrm{B}$, and some overlapping meanings can be served by both constructions. When the speaker thinks of a meaning that lies in a non-overlapping area, there is no choice. The speaker has to pick the construction that expresses that nonoverlapping meaning. Every time they do that, the connection between the form and the non-overlapping meaning is strengthened, and the connection between the form and the overlapping meaning is punished, so that it is weakened a little bit. This is a natural explanation for why over time we see cases of semantic differentiation.

A constructional, link-based explanation thus supports the view that linguistic units can be in competition, and that this competition either leads to substitution or differentiation. This is the idea that De Smet and colleagues (2018: 197) have examined in their paper that I mentioned yesterday. Across different theoretical frameworks, differentiation can be viewed as the default consequence of constructions or linguistic forms being in competition. However, De Smet and colleagues notice that competition can also have the opposite effect. There can be attraction. That process is driven by analogy. Let me read a quote from De Smet et al. (2018: 197): "As a result of analogy, competing forms often show attraction, becoming functionally more (instead of less) alike". That is a somewhat paradoxical situation. When we have multiple constructions that are paradigmatically related, and they can become either more different or more similar. Attraction can maintain and increase functional overlap in language.

This relates to an idea that I mentioned earlier in Lecture \#2, namely the grammaticalization process of paradigmatization. When a new grammatical construction emerges, it tends to join an existing paradigm, and it tends to adapt its behavior to that paradigm. Let me give just two examples from the history of English.

Indefinite determiners like $a$ and $a$ derive from a numeral word, one. The numeral one did not always belong to the paradigm of English articles. English did not always have an indefinite article, but in synchronic language use, the indefinite article has become a part of the article paradigm of English, contrasting with the definite determiner the. It has formed a little group of elements that serve the same grammatical function and do so by contrasting with each other.

The second example is more recent, i.e. the discourse marker now. Often when you will hear me say "Now, there is this problem", that now does not mean literally at this moment, but it indicates a shift of topic. The discourse marker 
now developed out of an adverb meaning at this time, and in present-day language use, the discourse marker now behaves like other discourse markers like well, or you know, or okay, that I could use alternatively when I am coming to a new topic. I could say, "Okay, now let's move on to this".

The underlying question is why constructions arrange themselves in paradigms. One explanation that has been proposed is that paradigms can be viewed as constructions themselves, as generalizations over generalizations.

This brings me back to Controversy \#2 from the first lecture. There are researchers that argue for constructions at a very high level of abstraction, so called higher-order schemas, in which speakers represent generalizations across abstract patterns. One example of this would be the dative alternation. If speakers form a generalization across John gave Mary the book and John gave the book to Mary, then they have a generalization that essentially constitutes a paradigm, a small paradigm in this case, as there are only two members. My main question for this lecture is the following. How do differentiation and attraction work in cases where we have paradigms of constructions?

In order to investigate that question, I brought another case study from my 2013 book, in which I look at a syntactic clausal pattern. The pattern involves a paradigm of forms that I call concessive parentheticals. They are illustrated by examples such as the ones that you see here, (1) Power, while important, is not everything, (2) It is an earnest, if unsophisticated, film, and (3) Although a Democrat, he has strong Republican support.

In this lecture, I want to introduce you to these constructions. I want to show how they have developed over the past 150 years, and I want to demonstrate how the analysis of these constructions speaks to the issue of these abstract representations of groups of constructions as a higher-order schema.

Concessive parentheticals can be defined in terms of several features. First of all, they contain a concessive subordination conjunction, which can be instantiated by elements such as while in "Power, while important, is not everything", if in "It is an earnest, if unsophisticated, film", and although in "Although a Democrat, he has strong Republican support". Semantically, concessive clause relations cancel or reject a potential implicature or a potential conclusion that someone might draw. The example "Although a Democrat, he has strong Republican support" expresses that one might think that a Democrat will not be supported by Republicans, but that this conclusion is not valid in this case.

Second, concessive parentheticals have a predicative element. There are these words like important, unsophisticated, or in the third example, $a$ Democrat, which are qualities that are predicated over an entity. The phrase although a Democrat conveys that he is a democrat. That is a predication. 
The third feature is syntactic. Concessive parentheticals are embedded into a superordinate syntactic matrix structure. They have a host clause in which they appear. For example, the concessive parenthetical while important occurs inside the sentence Power is not everything.

That embedding explains why these constructions are parentheticals. A parenthetical is a linguistic structure that is put into brackets, that functions as an afterthought, as something that you could also prefix with besides. You'll often see me doing a gesture that indicates that I'm putting my words into brackets. In writing and orthography, there are often commas, and in fact this is sometimes called comma intonation. "Power, while important, is not everything". You hear me saying this with little pauses. In the last two lectures, we have seen that variation is crucial for constructional change, and that is no different in this lecture.

Concessive parentheticals exhibit variation with regard to the features I just mentioned. There is variation in the conjunctions. There are four different ones that can be used and that I will be studying, although, though, while and if.

There is the further variation in the positions in which these parentheticals can appear. They are not always in the middle. They can appear at the very front of an utterance. Although unorthodox, the logic here is simple. If you put it in the middle, it goes The logic here, although an orthodox, is simple. It can even appear at the end, The logic here is simple, although unorthodox. With regard to their position, these constructions are rather flexible.

There is a massive variation with regard to the syntactic categories of this predicative element that I have mentioned, ranging over adjectives as unorthodox, adverbs as reluctantly, noun phrases like a Democrat, prepositional phrases, past participles, and even entire clauses. There is a wide range of syntactic variation.

Finally, there is another type of syntactic variation that concerns the embedding of the concessive parentheticals in their matrix structure. Concessive parentheticals can be embedded at the level of the clause. In the example "Power, while important, is not everything", while important is embedded in the clause Power is not everything. But there is a second way in which concessive parentheticals can be embedded, which would be at the level of a phrase, for example, a noun phrase. In "It is an earnest, if unsophisticated, film", the parenthetical appears inside a noun phrase: an earnest, if unsophisticated, film is a heavy noun phrase.

In summary, concessive parentheticals are headed by a concessive subordinating conjunction, they involve a predicative element, they are hosted by a matrix clause, and they vary with respect to their conjunction, their relative position in the clause and their internal syntactic structure. 
My first empirical question concerns the emergence of concessive parentheticals. How did concessive parentheticals constructionalize in the sense of Traugott and Trousdale (2013)? There are two possible hypotheses that I would like to explore. Hypothesis A would be that concessive parentheticals came about through a process I have mentioned before, namely reduction. Reduction commonly occurs in grammaticalization. Phonological reduction is a frequent phenomenon, but reduction can also be observed on the syntactic level. Hypothesis A would state that concessive parentheticals have come about through reduction of a full concessive clause. Some of the words are left out, and we are left with a more compact structure.

Hypothesis B would posit that concessive parentheticals came about through analogy from other clause types. For example, if we take temporal subordinate clauses, we know that they have reduced variants as well. In English, you can say things like "While young, swans are actually grey". I do not know if you've ever seen baby swans. When they hatch, they look like grey ducks, and then they grow and shed their grey feathers, and they end up white. The phrase "while young" thus means "during the time that they are young". Since this exists as a temporal subordinate clause structure, why not simply analogize that and form concessive clause that work more or less in the same way? While young, Reed is rated as a top lawyer, that does not mean "While he is young, Reed is a good lawyer", but rather "although he is young". You expect young lawyers to be less experienced and not as good. The example counters this conclusion.

Both reduction and analogy are recognized as forces that shape language change. With regard to parenthetical structures, reduction is more commonly invoked than analogy. If we have an elliptical phrase pattern, it is quite natural to think that it is the result of reduction, where speakers cut corners and shave down an expression to a more economic form.

Reduction is of course ubiquitous in language change. This has been expressed, for example in Givón's (1971) slogan “Today's morphology is yesterday's syntax". But crucially, reduction is not the only game in town. Fischer (2007) has shown that the default assumption of reduction processes in syntax can lead to faulty conclusions. Brinton (2008) comes to the same conclusion. Brinton (2008) has investigated parentheticals in English, structures such as $I$ mean, I find, or you see, and she finds that diachronic corpus data lends little to no empirical support to the reduction hypothesis. That means with regard to concessive parentheticals, we should also be very careful before we assume reduction as the most plausible explanation.

There are further problems for reduction, because there are some examples of concessive parentheticals that you simply cannot expand into a full clause. Let me just present one example here. Hood is a seasoned though disillusioned 
diplomat. It is not possible to expand that example into *Hood is a seasoned, though he is a disillusioned diplomat. That yields an ungrammatical sentence. Concessive parentheticals that are embedded at the phrase level do not allow an expansion of this kind.

How can we find out whether reduction or analogy is more likely? Again, I would like to use corpus data to examine that question. I want to operationalize the structural features of these constructions in such a way that their corpus frequencies can inform the controversy between reduction and analogy, and I would like to weigh the relative likelihoods of the two scenarios.

For the analysis, I used the TIME corpus of American English, which consists of journalistic writing. Why did I do that? Concessive parenthetical constructions are at home in elegant journalistic writing. It is a very compact structure. It is one that allows you to convey a lot of content in relatively little space, and that is what journalists need to do. My data comprises two concessive conjunctions, namely although and though. I also investigated two polysemous conjunctions namely, if and while. The main function of if is its use as a conditional conjunction. Its concessive function is merely secondary. The same is true for while, which is mainly a temporal conjunction, not a concessive one. I took random samples of 5000 concordance lines for each conjunction and then manually identified target examples. Most of the examples for if and while were of course conditional and temporal, not concessive.

Four types of examples

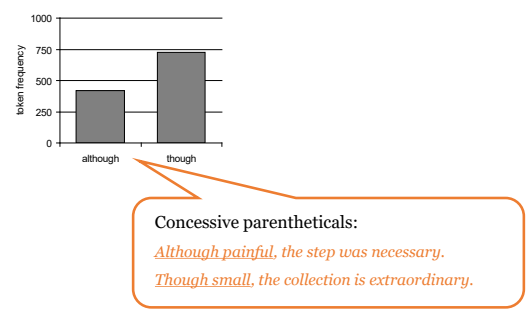

FIGURE 1

Let me talk about the kind of data I was working with. Overall there are four different types of example. The first type includes concessive parentheticals with the conjunctions although and though. In this graph that you see that I obtained about 400 examples of concessive parentheticals with although, such as, "Although painful, the step was necessary", or "Though small, the collection is extraordinary". You see the token frequencies of those constructions. 


\section{Four types of examples}
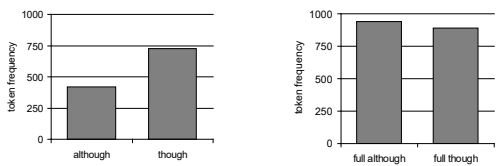

Full concessive clauses:

Although it was painful, the step was necessary.

Though the collection is small, it is extraordinary.

FIGURE 2

The second type are full concessive clauses with although and though. I obtained about 800 examples for each category with although being illustrated by examples such as "Although it was painful, the step was necessary", or "Though the collection is small, it is extraordinary".

\section{Four types of examples}

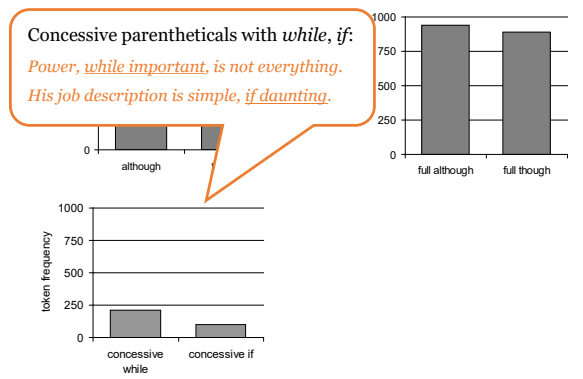

FIGURE 3

Moving on to the third type, here we have concessive parentheticals with two other conjunctions, namely while and if. You see that these types are less frequent. They are illustrated by "Power, while important, is not everything" or "His job description is simple, if daunting".

The fourth and final type are temporal and conditional parentheticals with while and if, which are again quite frequent. Temporal while-parentheticals are illustrated by "He was driving, while under the influence". A conditional example with if would be "If possible, patients should be treated at home". These are the four sources of data that I used. Why these four types? That relates to the two hypotheses that I wanted to test. 
Four types of examples

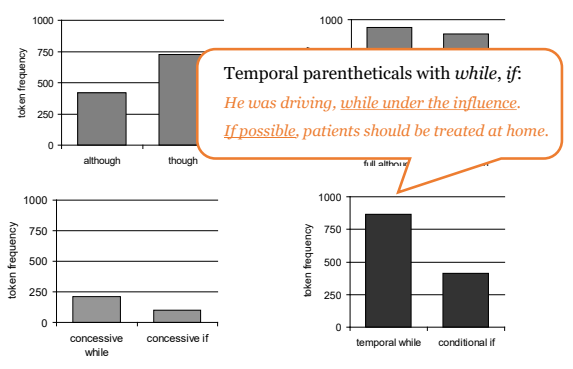

FIGURE 4

Four types of examples

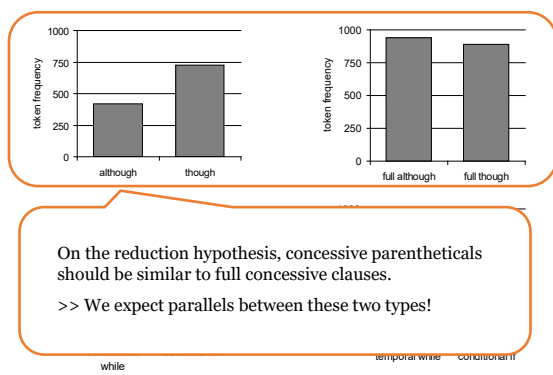

FIGURE 5

On the reduction hypothesis, concessive parentheticals derive from full concessive clauses, so these two types should be similar to each other. We expect parallels between these two in terms of their structural features and in terms of the lexical items that occur within them.

By contrast, on the analogy hypothesis, concessive parentheticals should be similar to temporal or conditional parentheticals. We would expect parallels between these two types. Concessive parentheticals with while should be similar to temporal parentheticals with while. Concessive parentheticals with if should be similar to conditional parentheticals with if.

I annotated all examples in the database for the relative position of the subordinate clause. Is the subordinate clause initial, medial, or final? I also annotated the syntactic structure of the predicative element in the subordinate clause. Is that predicative element an adjective, a noun or something else? I further annotated the examples for their lexical types. This means that I noted the adjective small in although small, or the adjective necessary in if necessary. 


\section{Four types of examples}

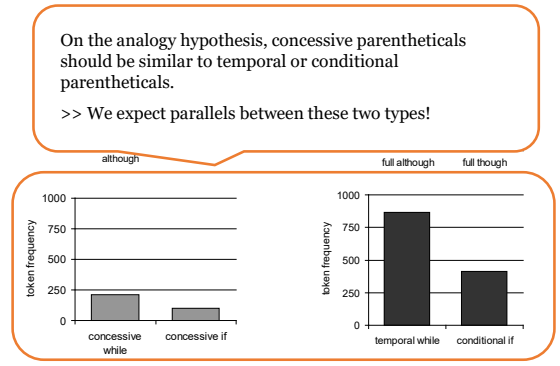

FIGURE 6

In order to perform quantitative comparisons, I used chi-squared tests of independence. Let's talk about while first.

On the analogy hypothesis, temporal parentheticals give rise to concessive parentheticals, and I mentioned the parallelism between "While young, swans are actually grey" and "While young, Reed is rated as a top lawyer". This would predict that temporal and concessive examples with while would be similar in terms of their preferences for their position, syntactic structures and lexical collocates.

while: differences in position

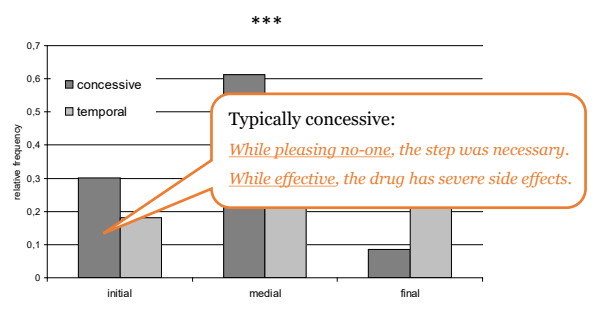

FIGURE 7

Is this the case? The short answer is no. When we compare temporal and concessive uses of while, there are significant differences with regard to their syntactic positions. Parentheticals with initial while are typically concessive, as in While pleasing no-one, the step was necessary or While effective, the drug has severe side effects. 
while: differences in position

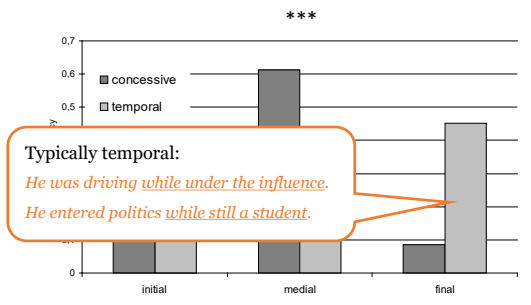

FIGURE 8

If we look at examples with final while, the relative preferences are very different. Final parentheticals typically have temporal meaning, as in He was driving, while under the influence or He entered politics while still a student.

while: differences in syntax

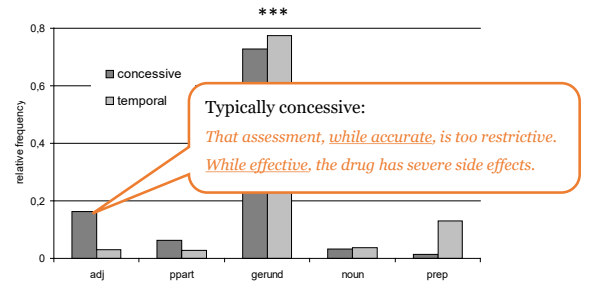

FIGURE 9

I observed further differences with regard to the syntax of the predicative element. In the category of adjectives, there is a relative preference for concessive meaning. This is illustrated by examples such as That assessment, while accurate, is too restrictive or While effective, the drug has severe side effects.

At the other end of the graph, there are prepositional phrases, which are preferentially used with temporal meaning, as in He was driving while under the influence or He met her while at Harvard.

I further examined the lexical words that appear in these constructions. For temporal and concessive while, there is practically no collocational overlap. They do not occur with the same word types. Concessive while occurs with adjectives such as accurate, or agreeable, or beautiful. Temporal while is used 
while: differences in syntax

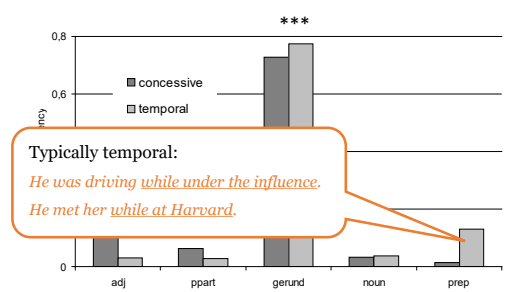

FIGURE 10

with adjectives such as alive, asleep, or drunk. There is no overlap. That suggests that it is rather unlikely that one developed out of the other. If we had this natural relation between them, we would expect some remnants of overlap.

In summary, it is unlikely that concessive parentheticals with while emerged as an analogy to temporal while-clauses, because they differ in terms of their syntactic placement, the structure of the predicative element, and their collocates. That is a negative result for the analogy hypothesis.

Let's look at if. On the analogy hypothesis, concessive parentheticals with if should derive from conditional parentheticals. Conditional parentheticals such as "If successful in the semi-final, they will play against the Netherlands", should give rise to concessive parentheticals, such as "If successful in the semi-final, they still lost against the Netherlands". That last example means that although they were successful in the semi-final, they still lost against Netherlands. As before, with while we observe differences rather than similarities with regard to the same parameters.

if: differences in position

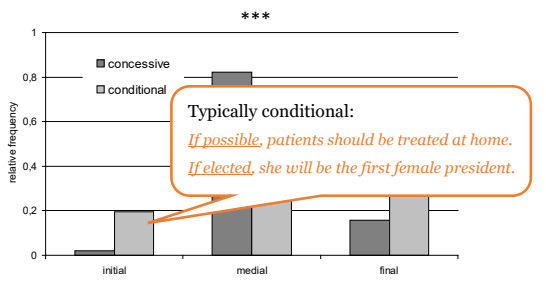

FIGURE 11 
Here you see the differences in syntactic position. The graph shows differences in each category, initial, medial, and final. Let me just talk about the initial position here. Initial if is typically used with conditional meaning, as in "If possible, patients should be treated at home" and "If elected, she will be the first female president".

\section{if: differences in position}

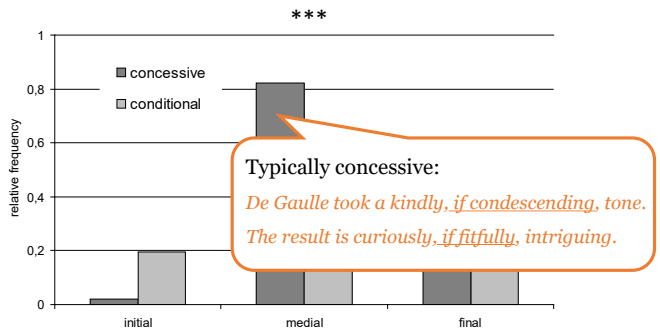

FIGURE 12

By contrast, medial parentheticals are typically concessive, at least they have a much higher preference for concessive meaning. Let me just take the second example, "The result is curiously, if fitfully, intriguing".

Let's look at the predicative elements. Also here, the pairs of bars have very different heights. Especially past participles are a strong cue for conditional meaning, as in He will face a life sentence, if convicted, or If elected, she will be the first female president.

By contrast, -ing clauses are typically concessive. An example would be His job description is simple, if daunting.

if: differences in syntax

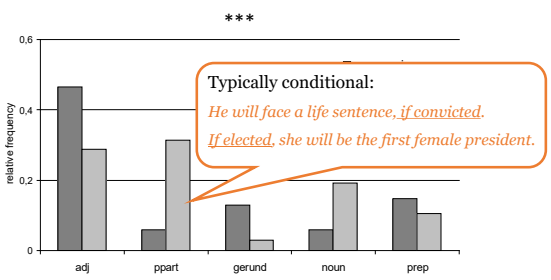

FIGURE 13 


\section{if: differences in syntax}

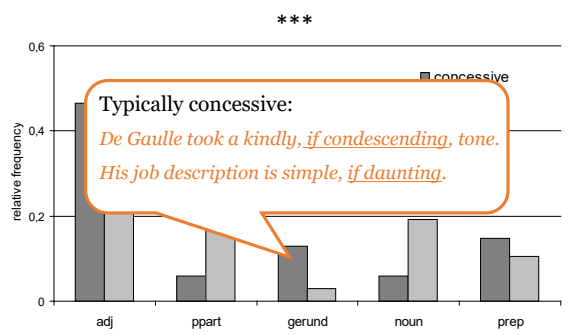

FIGURE 14

In terms of collocates, we see almost no overlap between conditional if and concessive if. There is one exception to that tendency, namely the adjective accurate. In If accurate, the results are sensational, the meaning is conditional. On the condition that his results are accurate, then this is great news. But in the second example, we have concessive meaning: It was an amusing, if not fully accurate, report of his activities. Besides that, concessive if and conditional if occur with very different sets of adjectives.

In conclusion, the evidence suggests that concessive parentheticals with if did not emerge as an analogy to conditional if-clauses, because there are simply too many differences across the structural and collocational parameters.

That leaves although and though. Let's move on to them. Here the data allows us to test the reduction hypothesis, which would predict that concessive parentheticals derive from full concessive clauses. Examples such as Although he is a Democrat, he has strong Republican support should, over time, give rise to Although a Democrat, he has strong Republican support. More specifically, full and parenthetical examples with although and though should be similar in terms of their syntactic structures and the lexical collocates. That is what we are going to look at next.

This slide presents the syntactic properties of full clauses in light grey and parentheticals in dark grey. You see that there are some differences, but overall the pairs of bars are more in line with each other than in the graphs that we saw with if and while.

This graph shows the syntactic categories for though. Again, the results are fairly similar. A chi-squared test yields the result that the differences are significant, but in comparison with if and while, these differences are minor.

In terms of collocates, there is much more overlap between full clauses and parentheticals than what we observed earlier with if and while, namely 


\section{although: very similar syntax}

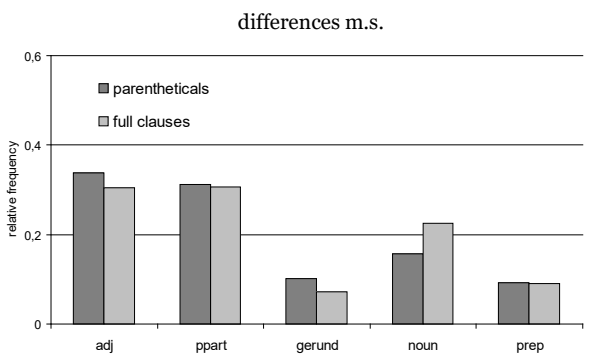

\section{though: fairly similar syntax}

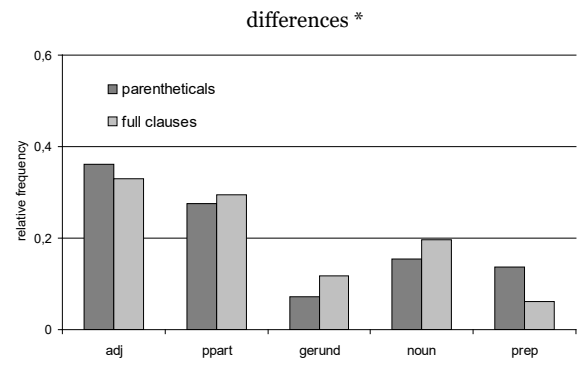

FIGURE 16

about $10.5 \%$. You might say that this is not very much. That is true, but it is definitely more than what we saw earlier. In conclusion, the corpus data are largely congruent with the reduction hypothesis. Concessive parentheticals and full concessive clauses have relatively similar syntactic structures and they have some collocational overlap. When we compare though and although, it seems that though-parentheticals are a little bit more different from their full counterparts than this is the case for although. They seem to lead the way in this differentiation process. If you imagine that we have a paradigm that is emerging, with every construction variant trying to find its niche where it serves a particular function, then though is in the vanguard, and it has emancipated itself to a stronger degree from the full clauses than its counterpart with although.

Let me come to a close with regard to the issue of reduction or analogy. In the analysis of parenthetical, elliptical, or otherwise apparently reduced 
structures, the process of syntactic reduction should not be assumed as a default. I take that idea from Fischer (2007) and Brinton (2008). However, in the case of concessive parentheticals with though and although, the reduction hypothesis holds up rather well. We have some structural evidence, and we have collocational evidence. All of that makes me confident to posit a relation between full and parenthetical clauses. The alternative hypothesis of analogical change from temporal and conditional parentheticals is not at all supported by the corpus evidence.

I'd like to come back to De Smet and colleagues (2018) and their observation that analogy has an important part to play in the mutual attraction of constructions. I want to do so by focusing on the question of whether concessive parentheticals become more similar or more differentiated over time. I have mentioned the idea that we observe a paradigm that comes into being, and it merits some consideration to examine what analogy may have to do with it.

I have mentioned that concessive parentheticals show a fair amount of variation across a range of several variables. I have also suggested that concessive parentheticals can be seen as a paradigm of constructions. Paradigms of constructions, as researchers like Florent Perek have recently argued, could be regarded as higher order schemas and as meta-generalizations that are represented at a very abstract level in the network of constructions. My question for the remaining time that I have here would be whether there is a general concessive parenthetical construction? Is there a construction that represents that generalization, that paradigm?

Let me specify that question a little bit more. How do we find out whether there is such a generalization? The generalization should correspond to a schema in speakers' minds that allows them to combine any kind of concessive conjunction, like although, though, if and while, with some kind of predicative phrase structure that can be an adjective, an adverb, a noun, a prepositional phrase, a past participle, -ing clause, and that can be embedded in matrix structures that are either a sentence or a noun phrase. If you think of that as a very general syntactic rule, a very productive schema that can generate many different concessive parentheticals, that would be the kind of generalization that speakers would have to have in their minds.

What would the evidence for such a high level generalization look like? You know that I like to work with diachronic data. I think that diachrony holds a cue with regard to this question here for us. If we see that the constructions of a paradigm structurally assimilate over time, we would have a reason to say that a meta-generalization is forming. If the conjunctions, for example, combine more and more freely with different types of predicative elements, and if the relative frequencies of structural variants becomes more and more 
homogeneous over time, that would be evidence for an increasingly paradigmatic structure.

Let me give you a hypothetical example. Let's say that adverbial parentheticals are at first primarily attested with although as in John apologized, although reluctantly. Let us further say that eventually such structures also appear with if and with while, so that speakers start saying "John apologized, if reluctantly" or "He apologized honestly, while grudgingly". Those examples are not corpus examples, but let's say that the structural profiles of these alternatives become more similar over time.

Whether different concessive parentheticals become more or less similar over time can of course be measured. Relative similarities between concessive parentheticals with although, though, if and while can be operationalized in terms of the relative frequencies of their structural variants. How often is although used with adjectival structures in the matrix clause? How frequent are structures that are embedded in a noun phrase? Those are these examples like an interesting, if problematic, idea. How do those frequencies compare to the other conjunctions?

\section{A dynamic visualization of language change}

- Use a corpus that represents identical kinds of text across multiple periods of time

- Select a phenomenon and create a visualization for each corpus period.
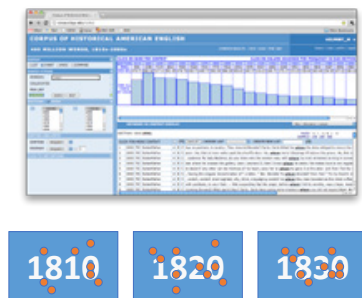

- View the visualizations in sequence.

FIGURE 17

At this point, I want to take a small step back to explain to you the methodology that I applied in this study. In order to see whether concessive parentheticals become more similar or more differentiated, I used a dynamic visualization of language change. The idea with that is that you use a corpus that represents identical kinds of text across periods of time. Then, you select a phenomenon and create a visualization for each corpus period. Then you view the visualization in sequence. Let me give an example of how this works in practice. Let's 


\section{Five verbs compared}

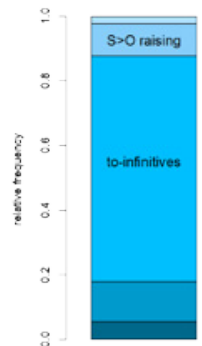

expoct

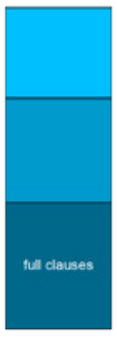

topose

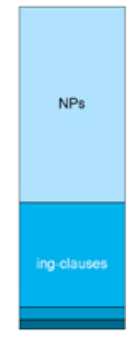

entory

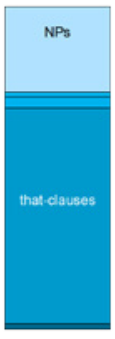

sumpst

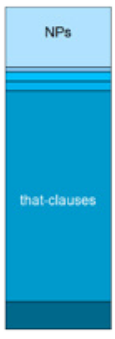

menson

FIGURE 18

take complement-taking verbs in English. Verbs such as expect, like, or imagine project a syntactic complement structure that can take different shapes. I can start a sentence with I expect, and I can finish it with, for example, a noun phrase, as in I expect a visitor. I can finish it with a more complex infinitive structure, as in I expect to hear from John. I can have a that-clause, as in I expect that John will win. Other options include raising constructions, as in I expect John to do well on the exam.

The key point here is that these different subcategorization frames differ in their relative frequency. Every complement-taking verb has a certain unique profile.

In this graph, I have compared five different complement-taking verbs, namely expect, hope, enjoy, suggest and mention. The $y$-axis shows the relative frequencies of the different complementation patterns that I mentioned. The relative frequencies add up to a hundred percent. The verb expect largely occurs with to-infinitives. It sometimes occurs with raising constructions, as in I expect John to win. The verb hope often occurs with full clauses, as in I hope I can see you again very soon. The verb enjoy frequently occurs with noun phrases, as in I enjoyed the food or I enjoy talking to you. Suggest and mention, frequently occur with that-clauses and noun phrases, as in I suggest that we meet this afternoon, or I suggested the fish. The overall profiles are different, but some verbs are closer to each other than others. The complementation profiles of suggest and mention are fairly similar. The other verbs have more individual properties.

The data in the bar graph is the basis for another visualization that we are going to create. The relative frequencies from the bar graph are shown as 
From relative frequencies to numerical distances
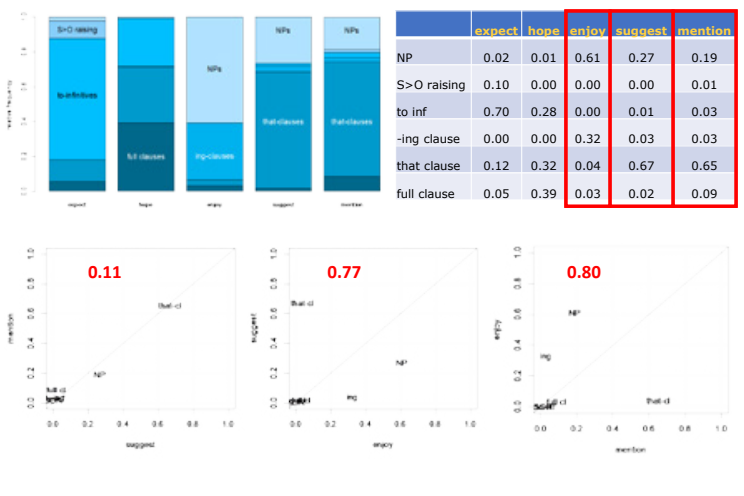

FIGURE 19

numbers in the table on the upper right. They can be used to calculate pairwise measures of similarity. For example, if you compare the relative frequencies of suggest and mention, you get a score of o.11 which indicates a high degree of similarity. When you line up the relative frequencies of suggest and mention and put them into a two-dimensional coordinate system, they form a nearly perfect diagonal.

By contrast, when you compare enjoy and suggest, you see that the relative frequencies are far away from the diagonal. Suggest frequently occurs with that-clauses. Enjoy frequently occurs with NP s. The two are not at all alike, and this results in a larger score of o.77. The same is true for enjoy and mention, which have very different preferences.

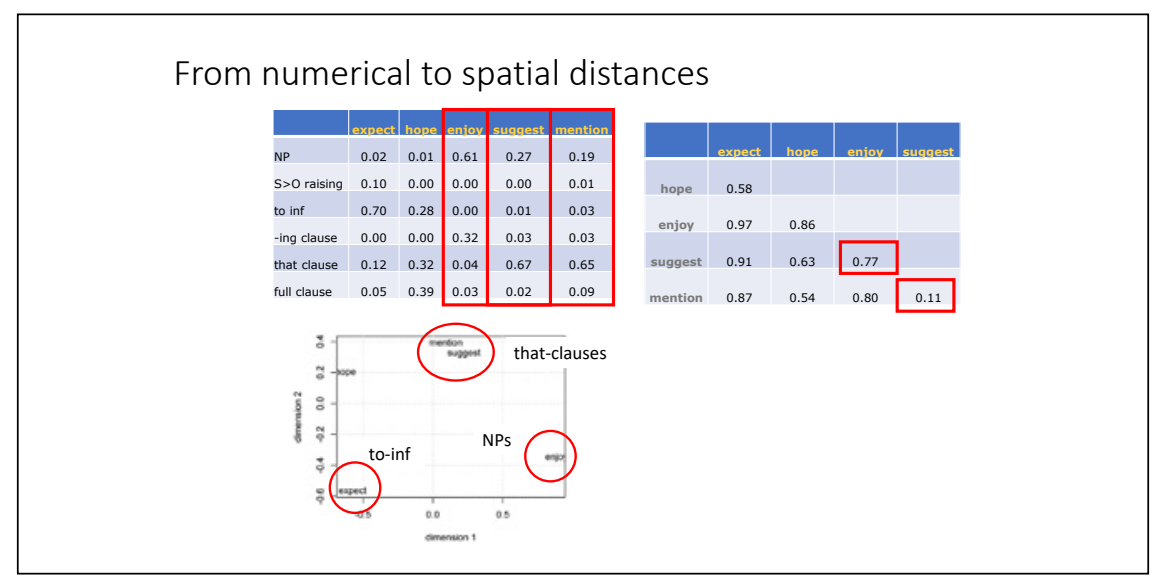

FIGURE 20 
Calculating measures of similarity for all possible pairs yields a distance matrix, which is what you see on the upper right of this slide. That kind of distance matrix can be transformed into a two-dimensional map that visualizes the distances. The verbs suggest and mention have a score of o.11. As a result, they pattern together in the map that you can see on this slide.

The verbs enjoy and suggest have a larger score of 0.77 , and they are farther away from each other in this representation. The different positions in the graph reflect the preferences of these verbs for different complementation patterns. Mention and suggest have a strong preference for that-clauses. Enjoy has a strong preference for N P s. Expect frequently occurs with to-infinitives.

We are now going from toy data to real data. In doing that, we are just expanding this kind of data set that you've just seen from 5 verbs to 45 verbs. I have taken them from the literature on English complement-taking verbs, including expect, like, try, suggest, mention, and so on and so forth. For each of these I retrieved frequencies from the Corpus of Historical American English, using data from the 186 os to the 2000 s. I extracted frequencies of the subcategorization frames, i.e. the syntactic options that I have shown you for the periods.

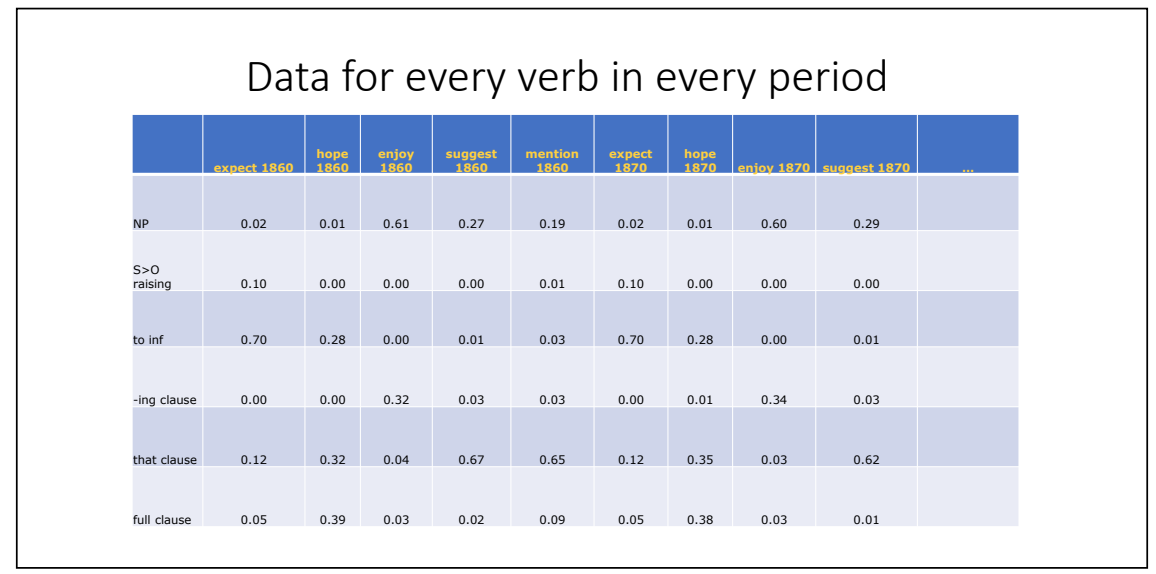

FIGURE 21

Proceeding in this way, I obtained data for every verb in every period. That means that the table that we are looking at now is a lot larger than what we had before. I am comparing expect in the 186os to hope in the 186os, to enjoy in the 186os, to suggest in the 186os, and so on and so forth. Expect from the 186os is also compared against expect from the 1870 , where the relative frequencies might have changed a little bit. Every verb is thus compared against all the others, and it is compared against itself in different time periods. 
We end up with a distance matrix that has $675 \times 675$ cells. We have 45 different verbs times 15 sequential periods. The distance matrix forms the basis for an explorative statistical analysis. One method that allows you to do that is called multi-dimensional scaling. That technique takes this distance matrix and aims to place all data points on a two-dimensional map, in such a way that the mutual distances are preserved as accurately as possible. This means that the method is going to cut corners. The representation is never perfect, but there are quality measures that are in place. The maps that you will see here meet common quality standards. Instead of viewing all data points at the same time in a single graph, we view them time-slice by time-slice in a movielike fashion.

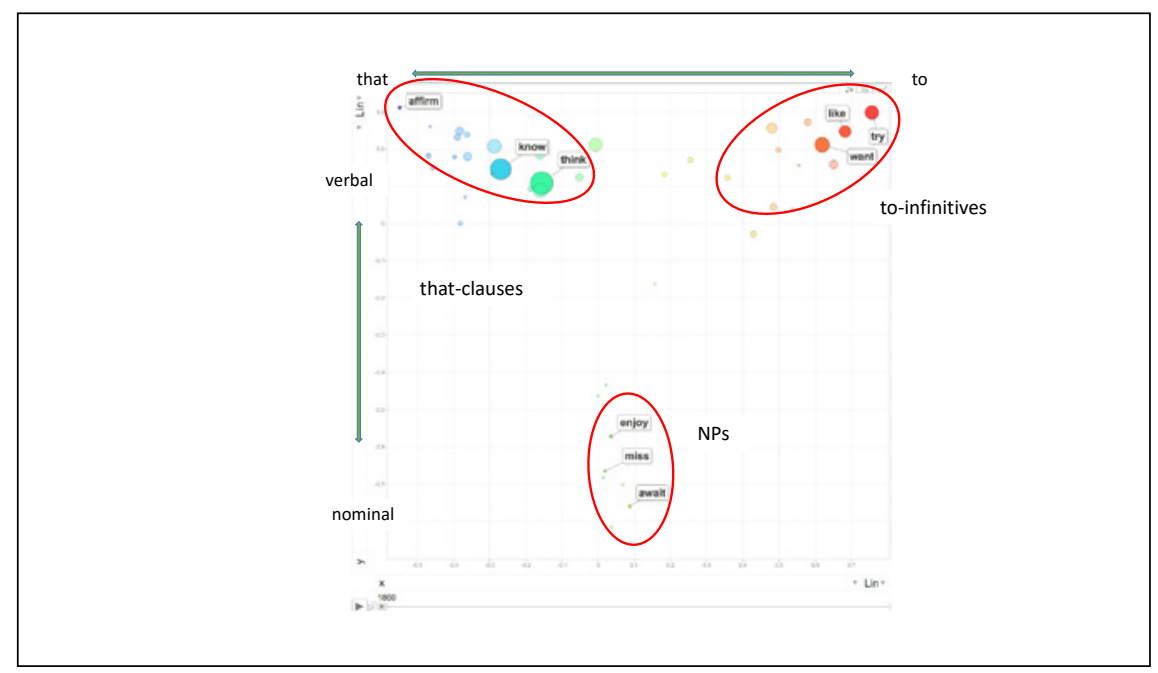

FIGURE 22

On this slide you see a map just like the one that I showed you earlier with expect, enjoy, and suggest, except that now we have all 45 verbs and we have analyzed them across the 15 different time slices of the сон data. On the basis of diachronic data, we can see how the configuration of verbs develops over time.

Over time, the complementation preferences of the different verbs undergo changes. The overall system in its triangular shape stays in place. That system shows clusters of verbs with different preferences. They are arranged in what we could call a paradigm. You see little points moving about, those are infrequent verbs for which the relative frequency measurements are not particularly reliable. The larger bubbles tend to stay in in their places, which shows that their profiles are stable. 
Let me give you a guide on how to interpret this map. This cluster in the upper left, with verbs like affirm, or know, or think, they frequently occur with that-clauses. The verbs in the upper rght corner, try, like, and want, they occur with to-infinitives. The verbs at the bottom of the graph tend to occur with NP s, i.e. enjoy, miss, and await. That does not mean that their preferences are absolute. Those are just preferences. Every single one of these verbs can potentially at least occur with all of the complementation patterns.

At the top, we have a continuum of preference from finite that-clauses on the left to non-finite $t o$-infinitives on the right. With regard to the $y$-axis, we have verbal complex structures at the top. We have nominal and more compact structures at the bottom.

Throughout the entire time that is analyzed, the two most important dimensions of verbal complementation are these two distinctions, the distinction between that-clauses and to-infinitives and the distinction between nominal and verbal complements.

Since this is just an illustration of the method, I will now move on and get back to concessive parentheticals. But before I do, there is one thing that I would like to show you, and that is the history of the verb confirm. Given all I have just said, confirm starts out in the 19th century as a verb that prefers noun phrases. Over time, confirm has drastically changed its complementation behavior. In the earlier decades it occurs with noun phrases, as in They confirmed the rumor, or They confirmed his position. By the 20oos, confirm is centrally in the cluster of verbs that tend to occur with that-clauses cluster. That is how the verb is used now, as in They confirmed that the story was accurate. Another interesting development concerns the verb dislike, which I have mentioned a couple of times in earlier lectures. You remember the frequency charts with dislike to do something and dislike doing something. These data actually reflect that same development. Dislike starts out in the to-infinitive cluster. As time goes on, it steadily but surely develops a preference for ing-clauses, which instantiate a nominal structure, i.e. gerunds, but which are nonetheless more verbal than just straight nominal phrases. That is why verbs that prefer ingclauses are situated in the middle of this whole configuration.

This was a fairly lengthy illustration of what this method does. I want us to get back to concessive parentheticals.

Let's look at the data for the concessive parentheticals, which look very similar to what we have just seen with the complement-taking verbs. Here we see historical data from the COHA that shows the syntactic variants of concessive parentheticals with although, though, if and while. Something that you see here is that all four conjunctions appear in parentheticals with adjectives. All of them have sizable black bars and the ratios are about the same. These are examples such as "Power, while important, is not everything". The one thing that 


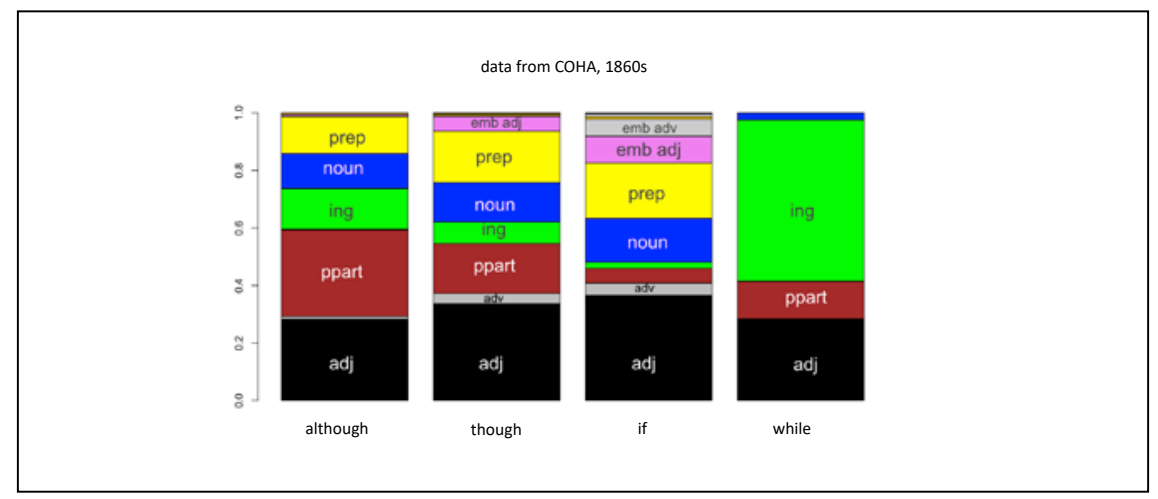

FIGURE 23

stands out in this graph is this large portion of -ing for while. The conjunction while has this strong preference for ing-clauses because of its temporal heritage. If, on the other hand, has the strongest preference for phrase-embedded examples. The part of the bar that is colored in pink represents examples such as "That is an interesting, if complicated, idea". You see that although and though are relatively similar in their distribution. Though has a little more of the phrase-embedded structures, and it has fewer examples of -ing than although. Although has fewer past participles and a few more adverbs.

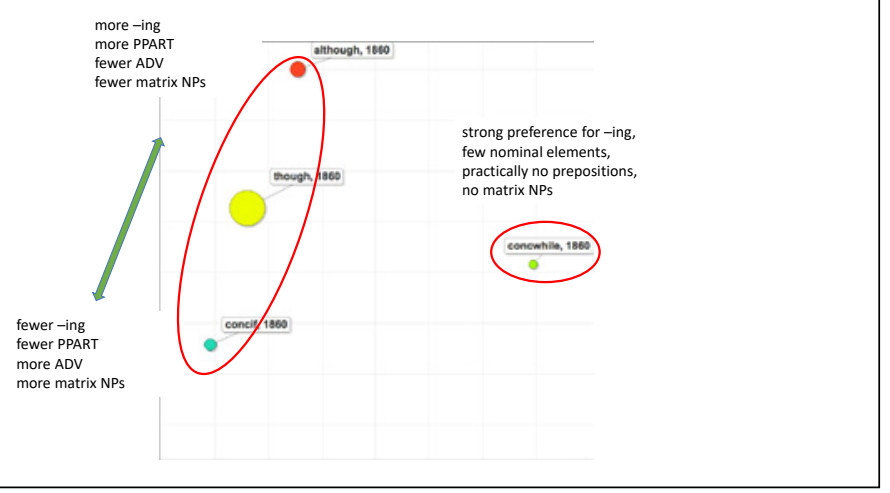

FIGURE 24

I transformed this data with multi-dimensional scaling in order to create the graph you see on this slide. The distances between although, though, if, and while reflect mutual similarity. This graph is based data from the 186 os.

Let's examine it in detail. While has a strong preference for-ing, it has few nominal elements, practically no prepositional phrases and no matrix NPs. The left side of the graph shows although, though, and if arranged on a cline that we 
can interpret. Although has more ing-forms than if, more past participles, fewer adverbs and fewer matrix NPs. Conversely, if has the fewest ing-forms in the set, it has the fewest past participles, it has more adverbs and more matrix NP s.

How has all of this has changed over time? Have concessive parentheticals become more similar over time, or have they become more different? What I hypothesized when I started this research was that concessive parentheticals would indeed converge on a common behavioral profile. I thought that over time, they would become more similar and gravitate towards the middle of the graph. Let's see what happens in reality.

If we look at the diachronic developments, we see that though and although converge on a similar pattern. By contrast, if and while move towards the outer margins of the graph. We see assimilation for although and though and differentiation for if and while. That means that a developing paradigm may incorporate conflicting processes. We can find two pairs of the paradigm matching up and functioning as near-synonyms that you can analogize to each other. And there are others that specialize in different functions.

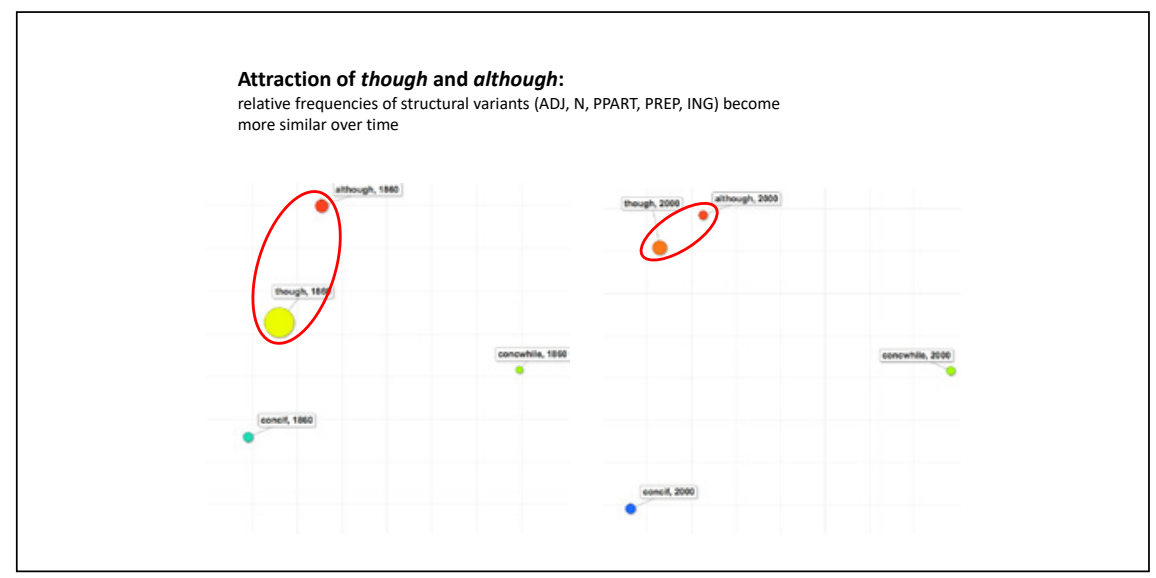

FIGURE 25

This slide shows the attraction of though and although. The left panel shows the graph from the 186os, and on the right you see the 200os, where though and although have clearly become more closely associated.

At the same time, though and if have been dissimilating. They are rather close to each other in the 186os, but they become more different over time. There is an outward movement of while. This slide shows where it started out in the 186os and where it ended up in the 200os. The same is true of $i f$, which moves further towards the bottom of the graph. 


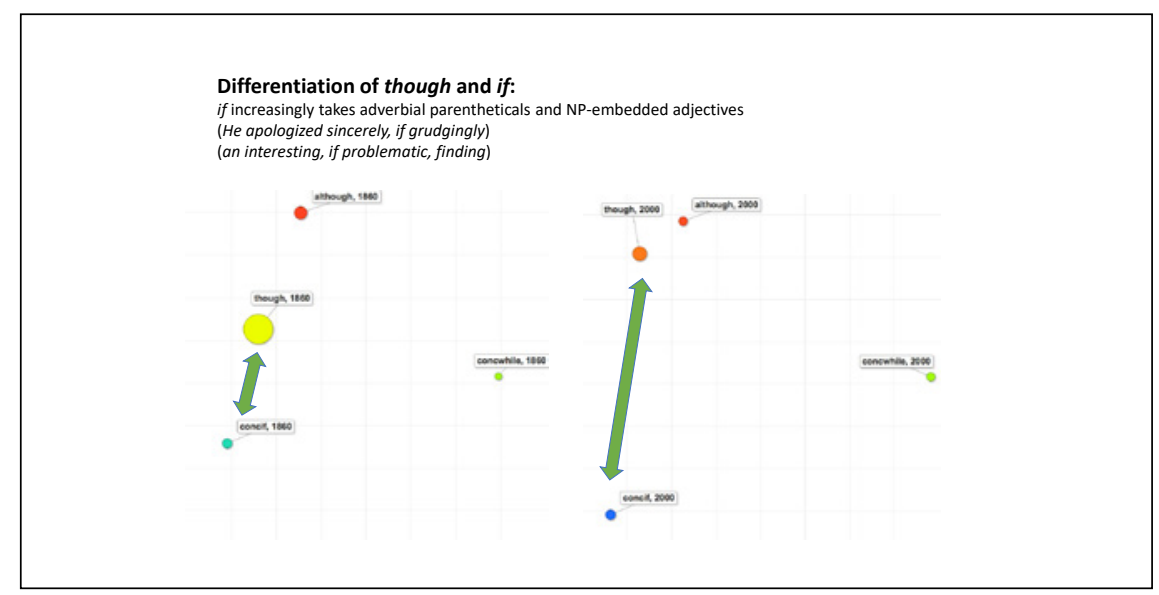

FIGURE 26

Outward movement of while:

dominance of ing-forms increases, adjectives decrease

FIGURE 27

To come to an interpretation of this development, between 1860 and 2008, there is no evidence to suggest that a general concessive parenthetical construction emerges. I would claim that for this construction family, it is unlikely that there is an overarching, higher order schema that would allow speakers to produce all kinds of variations of that construction. Rather, I think the local generalizations have become increasingly more important.

We observe processes of structural dissimilation with if and while, but within the overall process, although and though converge on a mutual constructional schema. I have been using the term paradigm for that idea. There is one other term that I think is useful in this context, and that would be the term of a construction family. A construction family is a set of constructions that share some resemblances, but that are not necessarily all connected to each other in the same way. 
To sum up, developments such as the ones that can be observed with concessive parentheticals illustrate what I wanted to express with the slogan "grammatical change is not a zero-sum game".

Paradigmatization is what we could call the constructionalization of a higher order schema. Within paradigms, partial functional overlap is actually common, not rare. Grammatical constructions may of course arrange themselves in a complementary distribution in functional space. That is something that is true not only of the data that you have seen here, but also of grammatical paradigms in general, across a wide range of languages. But that said, grammatical constructions may also inhabit the same functional space where they would then serve as variants of each other, and presumably stand in mutual competition, as I discussed yesterday with the case of my and myne.

I hope I have given you some ideas on how competition, differentiation and attraction are interrelated and how they are all necessary to understand what happens when groups of constructions change in the constructional network. I have also tried to argue that it is important to try to understand all of this in terms of links rather than in terms of constructional properties that are described directly into the nodes. With that I would like to end, and I thank you once more for your attention. 